\title{
An index for multidimensional assessment of swine health
}

\author{
Aidé Zavala-Cortés ${ }^{1}$ [D $\cdot$ Gerardo Hernández ${ }^{2} \cdot$ José-Víctor Calderón-Salinas $^{3}$
}

Received: 2 June 2020 / Accepted: 22 December 2020 / Published online: 6 January 2021

(C) The Author(s), under exclusive licence to Springer Nature B.V. part of Springer Nature 2021

\begin{abstract}
Pork accounts for almost one-third of the meat consumed worldwide. Infectious diseases have a marked impact on pig production. Epidemiological indicators are considered the most useful criteria in decision-making; however, a health status assessment remains a challenge at the national and regional levels. This study proposes a health index including herd-losses, morbidity, fatality, and type of diseases, to rate the health situation in a region or country; it contributes to assessing the effectiveness of control, damage manifestation, and trends. It is a multidimensional index with a structure of triads and simple quantitative, semiquantitative, and qualitative expressions that use flexible and dynamics limits. With it, we analyzed twenty-one countries in 2005-2018, focusing on African swine fever, classical swine fever, foot-mouth-disease, and porcine respiratory and reproductive syndrome, diseases that caused $72 \%$ of the morbidity. Our multidimensional approach estimates farm, local, and regional impact from infectious agents and outbreaks, and apprises trends aiming to be useful to control measures, strategic actions, and animal health policies.
\end{abstract}

Keywords ASF · CSF · Disease assessment · FMD · Pig farming $\cdot$ PRRS

\section{Introduction}

Over the last 18 years, world swine stock has grown $8 \%$ Asia grew 39\% (FAOSTAT 2019) - mainly due to the increase of intensive farms with large herd size and high swine density (FAO 2007; Gilbert et al. 2015), which has promoted health risks with a rapid spread disease, farms depopulation, and zoonosis (Morilla 2003). Infectious diseases impacted pork production at a local and global scale. For example, African swine fever was reported in 60 African, Asian, and European countries, with more than 8.2 million losses (dead and culled animals) (OIE 2020). Porcine epidemic diarrhea virus (PED) affected China and the USA in 2013, with

Aidé Zavala-Cortés

azavala@cinvestav.mx

1 Program on Science, Technology and Society, Centro de Investigación y de Estudios Avanzados del Instituto Politécnico Nacional, Mexico City, Mexico

2 Section of Metodology of Science, Centro de Investigación y de Estudios Avanzados del Instituto Politécnico Nacional, Mexico City, Mexico

3 Biochemistry Department, Centro de Investigación y de Estudios Avanzados del Instituto Politécnico Nacional, Mexico City, Mexico consequences in $\sim 50 \%$ of US breeding herds and $\sim 7$ million death piglets (VanderWaal and Deen 2018). Porcine reproductive and respiratory syndrome (PRRS), previously considered the primary economically devastating disease in the swine industry worldwide (Charerntantanakul 2012; Vilalta et al. 2017), had outbreaks in tropical countries (OIE 2019).

In that sense, ailments' effect is region-bounded or spread to a large scale with devastating effects, which demand better damage assessments at local and regional levels. The decision-making and planning processes in animal production include health evaluation, from the farm (Vilalta et al. 2017) to national or regional conditions (Perry et al. 2001). Different methods offer useful information to a health indicator from specific perspectives (Dehove et al. 2012). Epidemiological tools for surveillance and control help reduce the diseases' impact (Hoinville et al. 2013), contribute to the detection and control of animal and zoonotic diseases (Bisdorff et al. 2017) to guarantee safe trade, support decision-making in animal health, and control strategies; it involves hazard identification, comparative description of the herd's state, crosssectional analysis, risk assessment, and the estimation of the epidemic and seasonal tendencies, aiming to reduce the negative impact of animal diseases on zoonoses and economy (Perry et al. 2001; Rushton and Gilbert 2016; Bisdorff et al. 2017; Chenais et al. 2017). Some works define clusters 
(Tousignant et al. 2015) or the disease dynamics to analyze spatial-temporal trends of specific diseases (Vilalta et al. 2017), losses appraisal on the different animal populations in the world (World Bank et al. 2011); and recently, there is a participatory epidemiology analysis of the importance of livestock diseases (Gizaw et al. 2020).

The present work proposes a multidimensional health index, including the epidemiological variables losses, morbidity, fatality, and diseases, integrating factors for temporal, spatial, and pathological monitoring. It offers a simple, dynamic, and integrative alternative to estimate the health status by disease impact and outbreaks in a national or regional context, with publicly available data, suggesting trends, and contributing to assessing control measures, strategic actions, and public policies.

\section{Materials and methods}

\section{Data source}

Epidemiological events notified to the World Organization for Animal Health (OIE) from 01-01-2005 to 12-31-2018, split into three periods: 2005-2008, 2009-2013, and 2014-2018, by the nineteen major producer countries $(>8.5$ million of heads, $\sim 85 \%$ of the world's meat production, in 2017): Brazil, Canada, China, Denmark, France, Germany, India, Italy, Japan, Mexico, Myanmar, Netherlands, Philippines, Poland, Republic of Korea, Russian Federation, Spain, the USA, and Viet Nam, and two countries with low production (Argentina and Chile). Epidemiological data were collected from WAHIS Interface of OIE and the Ministry of Agriculture and Rural Affairs (MOA) reports. Stock data were obtained from FAOSTAT.

Data comprised the following: disease manifestation (no animal impact, sub-clinical/clinical infection, and emerging disease) number of outbreaks, susceptible animals, cases, dead, and culled animals, outbreaks date, and types of epidemiological units (farms, backyards, villages, slaughterhouse, and livestock market), including domestic swine and notifiable diseases with the international impact (OIE 2019). We classified the number of outbreaks per epidemiological units into four categories $(<10,11-50,51-99$, and $>100)$ and included them in the databases for spatiotemporal clustering dynamics. These categories were also used in the qualitative data analysis to establish MLF-index thresholds and disease characterization.

\section{Data analysis and index}

To build the index, we considered herd-losses, morbidity, and fatality. To simplify both calculations and interpretation for the index, we trisected each of these variables into low $(a)$, mild (b), and critical (c) categories. Although the boundaries for these categories may vary according to the scale of analysis and the episode evolution, we use the following criteria for the present analysis. For herd-losses, based on World Bank et al. (2011), the thresholds for those categories are $0.01 \%$ and $0.25 \%$. Besides, we propose the two morbidity boundaries, $0.01 \%$ for acceptable and $0.02 \%$ for critical rates, obtained from statistical analysis of twenty-nine observations of reported outbreaks (2005-2018) (OIE 2019). To fractionate fatality, we pose two thresholds resulting in three categories. High morbidity that yields low herd-losses defined the boundary for low fatality, $0.01 / 0.02=0.5$, whereas low morbidity that translates to high herd-losses determined the threshold for critical fatality, $0.25 / 0.01=25$. Below the low fatality threshold, we define category $a$, above the critical fatality correspond to category $c$, and between those limits, the corresponding index is $b$.

Herd-losses and morbidity allow estimating disease damage in proportion to head numbers by country. The notation used to calculate herd-losses rates is as follows: $\frac{d}{s}, d$ represents all dead and culled animals of all reported diseases by country in each period, and $s$ is the total of the country's stock in the same period. We calculate morbidity as follows: $\frac{c}{s}$, where $c$ denotes all diagnosed cases for all diseases by country in each period. Fatality $(\mathrm{F}), \frac{d}{c}$, is an indicator of the severity potential of diseases in each country; that indicator is the slope of a line going through the origin and passing on a point in the morbidity (M)-herd-losses (L) plane. We will represent the three effect ranges, low, mild, and critical, for each variable, as $a, b$, and $c$ (Fig. 1a). The health status of a region or country will be labeled using the triad (M,L,F). Thus, a triad $(c, b, a)$ corresponds to a place with critical morbidity, mild herd-losses, and low fatality. The index derived from this analysis will be named the MLF-index, and it will merely be the sum of the triad's elements, $\mathrm{M}+\mathrm{L}+\mathrm{F}$.

The 17 possible combinations of the levels $a, b$, or $c$ in the triad's three positions appear in Fig.1b. Note that there are some restrictions. In the area defined by acceptable morbidity and herd-losses, $a$ for these two variables, fatality can attain three levels $a, b$, or $c$, resulting in three possible combinations $a a a, a a b$, or $a a c$. However, in the area comprising intermediate morbidity and acceptable herd-losses, the possibilities for fatality are only $a$ or $b$, which gives two possible combinations for the triad, baa and $b a b$.

Following the same rationale, we may infer all possible combinations (Table 1). To determine numerical values for $a, b$, and $c$, we simplify matters by establishing that the score index $(\mathrm{M}+\mathrm{L}+\mathrm{F})$ will only assume the values $0,0.2,0.4,0.6$, 0.8 , and 1 . These values represent the semi-quantitative form of MLF-index and correspond to qualitative impact values: null, low, low-medium, medium-high, high, and critical, respectively (Table 1). 
Fig. 1 Representation of morbidity (M), herd-losses (L), and fatality (F) limits. Dotted lines indicate morbidity and herdlosses thresholds (MLFacceptable and M'L'F'-critical). The slopes represent the acceptable (F) and critical (F') range of losses. a depicts acceptable ( $a$-white), medium ( $b$ light gray), and critical ( $c$-gray) areas. b Schematic representation of possible triads for acceptable $(a)$, medium $(b)$, and critical rates (c)
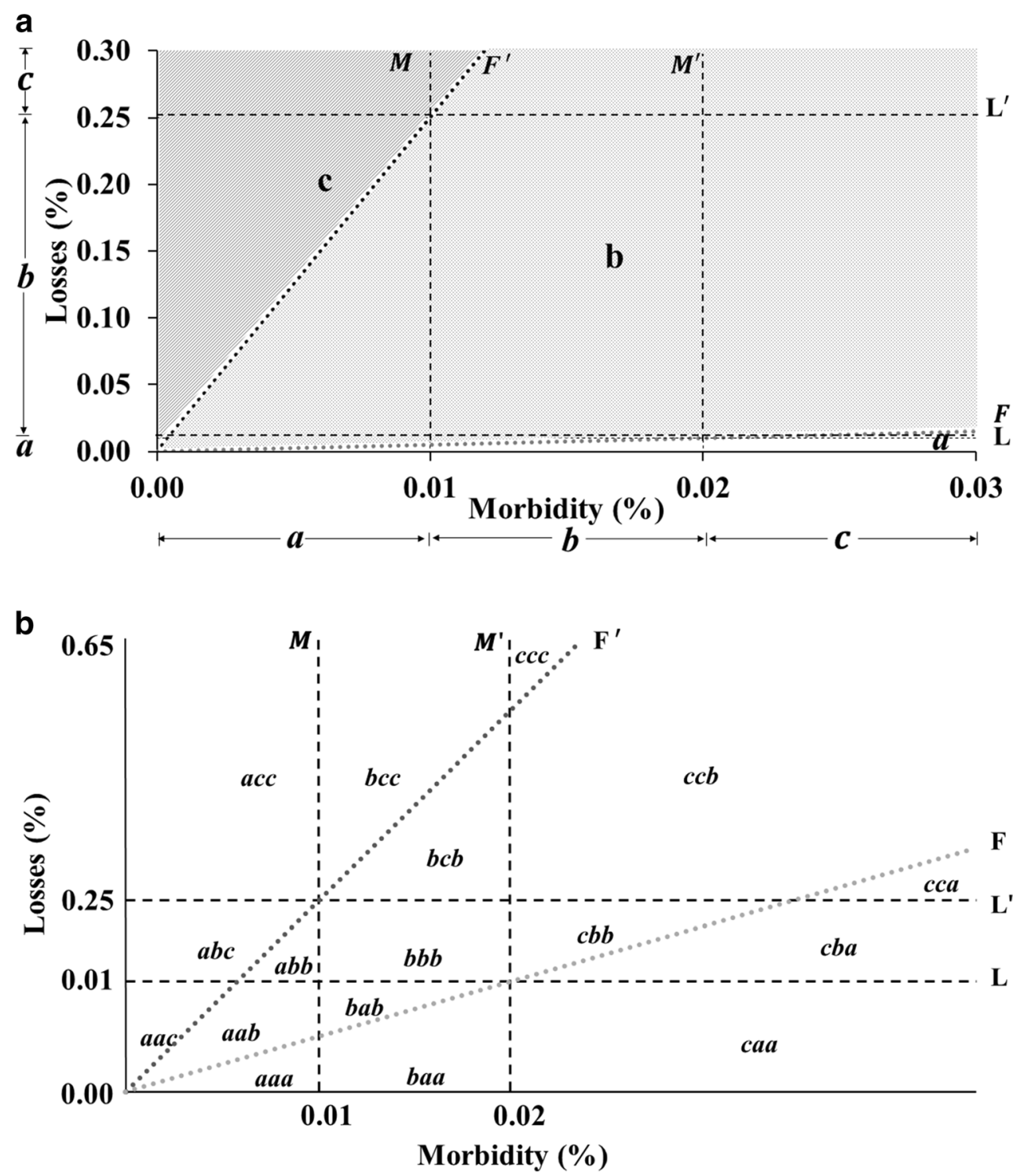

Since the sum of the three components of the triad ranges from 0 to 1 , we better assign 0 (null impact) to $a a a$, which implies that $a+a+a=0$, that is, $a=0$. Therefore, $a+a+b=$

Table 1 Score index and impact parameters in health status

\begin{tabular}{lll}
\hline MLF-index & Score & Impact \\
\hline$a a a$ & 0 & Null \\
$a a b, b a a$ & 0.2 & Low \\
$a b b, a a c, b a b, c a a$ & 0.4 & Low-medium \\
$b b b, a b c, c b a$ & 0.6 & Medium-high \\
$a c c, c c a, c b b, b c b$ & 0.8 & High \\
$b c c, c c b, c c c$ & 1 & Critical \\
\hline
\end{tabular}

Combinations follow the pattern MLF. Triad combinations, score values $(\mathrm{M}+\mathrm{L}+\mathrm{F}$, semi-quantitative), and impact (qualitative evaluation). Triad labels: $a$ acceptable, $b$ intermediate, and $c$ critical
0.2 , no matter the triad's order. We have then that $b=0.2$. To find the value of $c$, we accepted that a triad with $2 c$ 's and a $b$ ( $c c b$ and $b c c$ ) should be considered critical. That means that $c$ must have a value of no less than 0.4 . We chose this limit as its value, $c=0.4$. Though $c c c=1.2$, we set it at the maximum value 1 (Table 1$)$.

\section{Spatiotemporal distribution}

To compare the spatiotemporal distribution of diseases' analyses with the MLF-index, outbreaks data of the epidemiological units affected by ASF, CSF, FMD, and PRRS were processed using the statistical software R (R Core Team 2013), version 3.1.3, and package: Hmisc (Harrell et al. 2019), UsingR (Verzani 2018), rgdal (Bivand et al. 2019), and shapefiles (Stabler 2013). For spatiotemporal analysis, a map was made with GISTools (Brunsdon and Chen 2014), 
shapefile of the world map (www.arcgis.com/), and coordinates from (https://www.coordenadas-gps.com/) comprising the three studied continents: America, Asia, and Europe.

\section{Results}

Between 2005 and 2018, the 21 analyzed countries reported 16 different diseases: Aujeszky's, anthrax, brucellosis, cysticercosis, erysipelas, trichinellosis, African swine fever (ASF), classical swine fever (CSF), foot and mouth disease (FMD), influenza, Japanese encephalitis, novel enteric coronavirus, porcine reproductive and respiratory syndrome (PRRS), transmissible gastroenteritis, vesicular stomatitis, and porcine epidemic diarrhea. From 2005 to 2008, 6 diseases (204 outbreaks) were reported, 15 (1201) from 2009 to 2013, and 8 (1168) from 2014 to 2018, of which $72 \%$ of the cases were caused by four diseases; 1423 (55\%) occurred in commercial farms, 634 (25\%) in villages, and 506 (20\%) were backyard herds. Asia has the highest incidence, mainly caused by PRRS (512,000 cases/first period), though eventually reduced $(20,200 / \mathrm{s}$ period). FMD was the most prevalent in the third period $(173,364)$, followed by ASF $(124,000)$. In the American continent, the only relevant disease incidence was PRRS in the second period (2603), whereas in Europe, ASF had a high incidence (1339) in the third period (Fig. 2).

When comparing incidence and fatality, PRRS had more cases, but the fatality rate caused by this disease was less than two animals dead or killed for each sick animal. In contrast, ASF caused massive fatality with few animals diagnosed with the disease. FMD was the second disease with the highest fatality rate, while CSF had the lowest incidence and a low fatality ratio (Fig.3).

We adopt three impact categories to weigh the damage observed in hog farming diseases: "catastrophic" denoted as +++ , "marketable" as ++, and "normal" as +, following the pattern of risk management strategies and policies (OECD 2011). To categorize the different diseases in the above scheme, we used information from the classification of disease notifiable to the OIE (2019) and categories of emergency animal diseases of the Australian Animal Health Council (2016) (Table 2).

The epidemiological dynamics varies for each country. In Russia, the impact grew steadily due to continuous morbidity increments at constant medium fatality; the MLF-index changed $a b b / b b b / c c b$ until herd-losses reached critical levels when morbidity also attained maximum level (Table 3). China improved its initial grievous situation $(c b b)$ in the subsequent periods in different forms. In the second period, the reduction in fatality accounts for the change ( $\mathrm{caa}$ ), whereas in the third, the reduction in morbidity does not produce the expected effect due to the increment in fatality $(a b b)$. Mexico and
Germany were affected by a high fatality rate but low morbidity in the second period $(a a c)$; the herd-losses remained low, and eventually, the diseases were controlled. In the two cases above, the " $c$ " fatality level resulted from isolated cases, which hints at the index's sensitivity at low scale measurements.

The MLF-index also allows for making comparisons between diseases (Table 4). In the first period, PRRS had the highest impact, according to MLF-index $=c b b$, with critical morbidity, intermediate herd-losses, and fatality, resulting in a high impact. In the second period, FMD caused the largest number of dead $(500,000)$, which reach intermediate herdlosses $(0.018 \%)$, with medium-high impact and MLF-index = $a b c$, while ASF morbidity $(0.03 \%)$ and herd-losses $(0.4 \%)$ held critical impact with MLF-index $=c c b$.

The spatial-temporal analysis shows a broad distribution of FMD and ASF in Asia (Fig. 4a). China exhibited many but small clusters, while Russia, with a medium-high impact $(b b b)$, and Korea ( $a c c)$ had massive clusters; both countries reached super-spreads $>100$ EU, Korea with three catastrophic diseases, and Russia with four. CSF was the less spread disease with small clusters. In America, CSF was the more spread disease, in Mexico (aac/score $=0.4$, low-medium impact), and Brazil ( $a a b /$ score $=0.2$, low impact), with small clusters. ASF had a substantial dispersion in Poland $(a b b /$ score $=0.4$, low-medium impact with one catastrophic disease), with some $>100$ EU clusters. PRRS had the highest spread in the first period, broadly affecting Southeast China and Viet Nam, with small clusters in Russia and China (Fig. $4 \mathrm{~b}$ and $\mathrm{c})$.

Table 5 shows four disease alert categories based on the multidimensional assessment of the MLF-index and the epidemiological dynamics in each country. The first corresponds to secure health states, the second to preventive, the third to alert, and the fourth to generalized alarm.

\section{Discussion and conclusion}

This work develops and proposes an index based on available data of morbidity, herd-losses, and fatality, and it was applied to assess sixteen reported diseases by nineteen countries, contrasted with incidence and spatiotemporal analyses. According to the scale used, thresholds for acceptable and critical levels are (0.01 and 0.02) for morbidity, (0.01 and 0.25 ) for herd-losses, derived from them, and (0.5 and 25) for fatality. Relative to the scale of analysis or as conditions dictate, modifications of such numerical thresholds are possible, preserving the structure of the analysis. The index is flexible and adaptable to specific disease conditions, the analysis scale, and the event evolution. This index contributes to other disease assessments, involving probability complex models, 
Fig. 2 Incidence (cases) of ASF, African swine fever; CSF, classic swine fever; FMD, foot and mouth disease; PRRS, porcine respiratory and reproductive syndrome in (a) Asia and (b) America (AM) and Europe (EU), in the three periods. Data from WAHIS-OIE
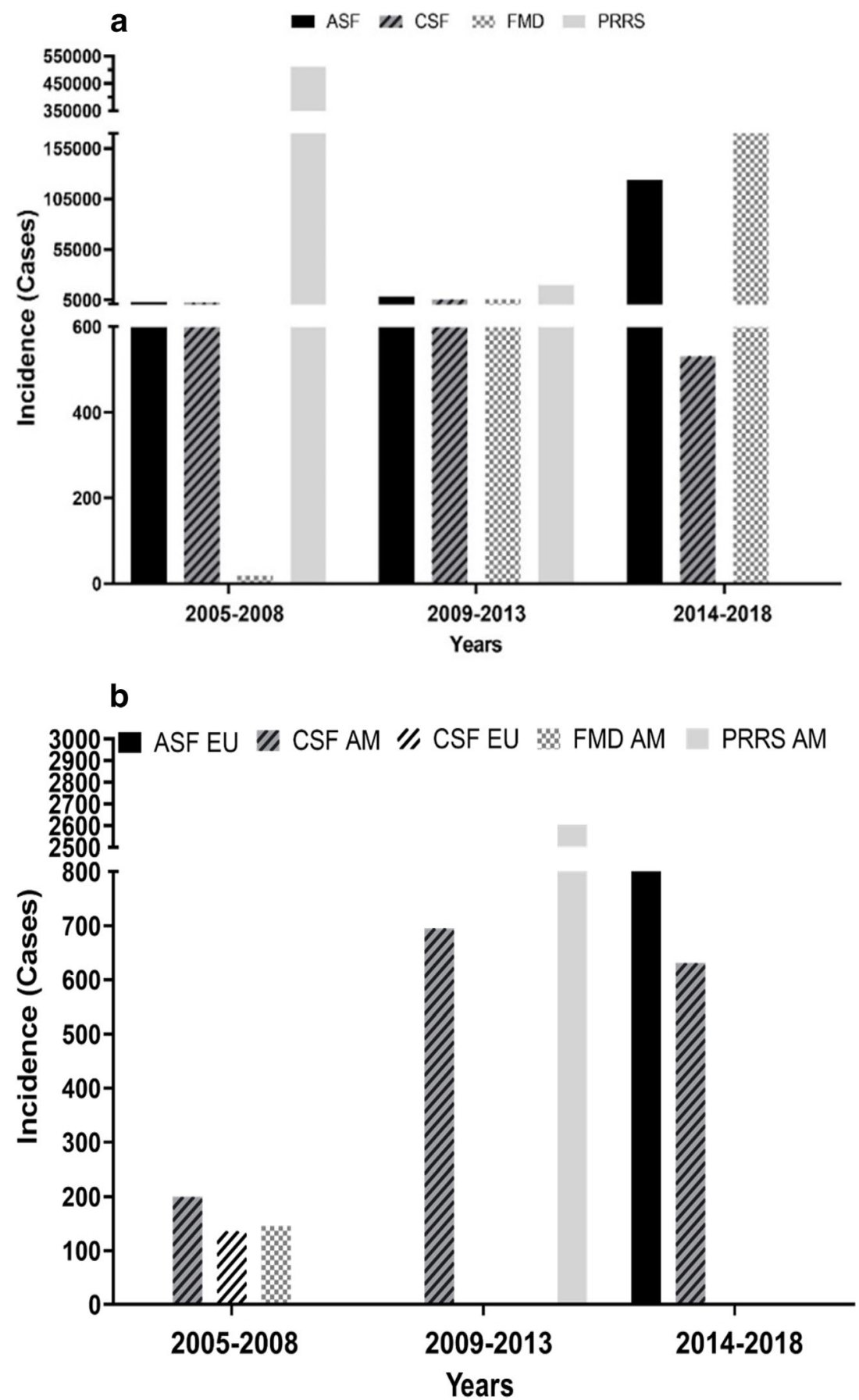

quantitative methodologies to measure mortality effect by livestock unit (LSU), and descriptive epidemiology patterns.

It is an alternative to studies on disease assessment that include surveillance activities, risk-based methods, losses quantification, economic estimations, spatiotemporal, and incidence analysis (Allepuz et al. 2007; World Bank et al. 2011; Hoinville et al. 2013; Holtkamp et al. 2013; Mur et al. 2014; Tousignant et al. 2015; Vilalta et al. 2017; Jurado et al. 2019).

OIE (2011) recommends systematic evaluations of risk with information about the pig products' origin, feedstuffs, hazards characteristics, animal movements, slaughter, and potential exposure routes of indigenous livestock. Participatory epidemiological studies based on the producers' perception provide another quite necessary perspective (Nthiwa et al. 2019; Gizaw et al. 2020). Our index requires readily available data and processes them in a simple form to produce quantitative, qualitative, and qualitative-quantitative labels (qualitative assessments are facile to compare to perception studies). The index simplicity makes it possible for producers and decision-makers to use the index, whereas complex stochastic 


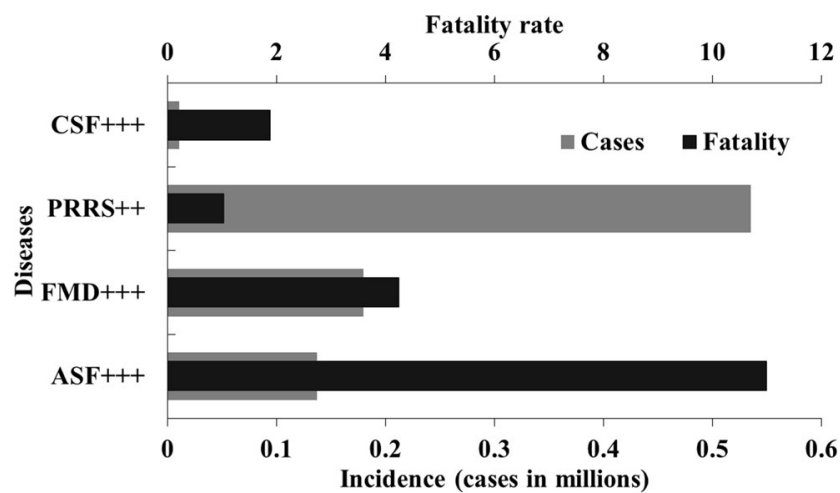

Fig. 3 Incidence (cases in millions) and fatality rate by disease in the twenty-one studied countries (2005-2018). ASF, African swine fever; CSF, classic swine fever; FMD, foot and mouth disease; PRRS, porcine respiratory and reproductive syndrome. Impact category: catastrophic $(+++)$, marketable $(++)$, and normal (+) diseases, according to the MLF-index. Data from WAHIS-OIE

and probabilistic models widely used in many different risk estimators (Mur et al. 2012; De la Torre et al. 2013; Jurado et al. 2019) are reserved to specialized groups. It attempts to be useful at different scales for temporal analysis, spatiotemporal distribution, health alert, and critical health states.

The index offered a broader perspective in the regional analysis to assess the complex disease's effect compared with incidence dynamics and fatality rate. On the continent scale, the incidence analysis of the four primary diseases allowed observing the changing aspects, but it did not show the

Table 2 Diseases impact classification

\begin{tabular}{lll}
\hline Pathogen & Impact & Assigned weigh \\
\hline ASF & Catastrophic & ++ \\
CSF & \\
FMD & \\
Influenza & \\
Trichinellosis & \\
Aujeszky's & Marketable & ++ \\
Anthrax & \\
Brucellosis & \\
Cysticercosis & \\
PRRS & \\
Vesicular stomatitis & \\
T. gastroenteritis & \\
Erysipelas & \\
Novel enteric coronavirus & \\
PED & \\
Japanese encephalitis &
\end{tabular}

Diseases impact classification according to, and modified of, OIE (2019), OCDE (2011), and Australian Animal Health Council (2016). Weigh is a semi-quantitative assignation of diseases impact. Semi-quantitative weight risk of the disease with highest impact: catastrophic $(+++)$, marketable (++), and normal (+) diseases diseases' full effect: in three of the four diseases, the fatalities were highest than incidence, evidencing the high impact on herd-losses. However, the fatality rate alone is not sufficient to assess the disease's effect, especially since it is impossible to observe the already documented considerable damage of PRRS (Zhou and Yang 2010; Holtkamp et al. 2013; Vilalta et al. 2017). This aspect may reflect a lack of reports, a contradiction in the data, or health management problems. However, the evaluation with the MLF-index makes evident the impact in temporal assessments by country or region. In the analysis by disease, the index is fair in evaluating PRRS and CSF impacts. Regarding solely the number of deaths, the most significant damage was due to FMD (second period), but the triad for FMD reflected only a medium-high impact (MLF-index $=a b c$ ) due to acceptable incidence but moderate fatality. On the other hand, ASF had the most devastating effect, with critical impact (MLF-index $=c c b)$, consistent with other findings (Sánchez-Vizcaíno et al. 2015). The number of deaths alone suggests that FMD was worse than ASF, but triplet analysis indicates that ASF was more damaging (second period).

Since both morbidity and herd-losses are values relative to the susceptible population, and fatality is an absolute value, their combination in the index provides different perspectives. In the second period in the regional assessment, the susceptible population was much larger considering China, Russia, Korea, and Japan, which results in a medium-high FMD impact $($ MLF-index $=a b c)$ but observing only the susceptible population in Korea and Japan, that are smaller, the disease impact is high (MLF-index=acc). In Korea's case, previous findings showed significant affectations of FMD (Jung-hyang 2019), with > 3.3million culled pigs (2010-2011) according to government reports (QIA 2014). However, the fatality rate is independent of the population size, and therefore, it is an intrinsic disease measure. Consequently, MLF-index gives a fair damage assessment by weighing three interrelated variables for a given population.

This approach is also useful to survey control measures to contain outbreaks, considering the four categories of alarm here presented. In FMD outbreaks, according to the data reported, Korea and Japan took drastic measures to contain the outbreaks, conducting cluster isolation at large; with a high fatality, isolation could lead to the prevention of further geographic dissemination, while in Russia, the ASF dissemination (since 2007) was higher and constant, with many clusters of various sizes, as previously noticed (Jung-hyang 2019; Perez et al. 2019). In Korea, two different conditions conduced to the "alarm" category for FMD. During the second period, the highest number of dead animals is seen in the critical herd-losses due to its excessive fatality, affecting 48 epidemiological units, with only 180 sick animals, resulting in a triad acc. In the third period, animal deaths decreased, but the incidence $(157,400)$ and the number of epidemiological 
Table 3 The MLF-index, weigh, and score in producers' countries in the three periods

\begin{tabular}{|c|c|c|c|c|c|c|c|c|c|c|c|c|}
\hline & \multicolumn{4}{|c|}{$2005-2008$} & \multicolumn{4}{|c|}{ 2009-2013 } & \multicolumn{4}{|c|}{ 2014-2018 } \\
\hline & MLF & $*$ & \# & Index & MLF & $*$ & \# & Index & MLF & $*$ & \# & Index \\
\hline Argentina & $a a a$ & & & 0 & $b a a$ & +++ & (1) & 0.2 & $a a b$ & +++ & (2) & 0.2 \\
\hline Brazil & $a a b$ & +++ & (2) & 0.2 & $a a b$ & +++ & (1) & 0.2 & $a a b$ & +++ & (1) & 0.2 \\
\hline Canada & $a a a$ & & & 0 & $a a b$ & +++ & (1) & 0.2 & $a a a$ & & & 0 \\
\hline Chile & $a a a$ & & & 0 & $b b b$ & ++ & (1) & 0.6 & $a a a$ & & & 0 \\
\hline China & $c b b$ & ++ & (1) & 0.8 & $c a a$ & +++ & (3) & 0.4 & $a b b$ & +++ & (2) & 0.4 \\
\hline Denmark & $a a a$ & & & 0 & $a a a$ & +++ & (1) & 0 & $a a a$ & & & 0 \\
\hline France & $a a a$ & & & 0 & $a a b$ & ++ & (1) & 0.2 & $a a b$ & ++ & (1) & 0.2 \\
\hline Germany & $a a b$ & +++ & (1) & 0.2 & $a a c$ & ++ & (1) & 0.4 & $a a a$ & & & 0 \\
\hline India & $a a a$ & & & 0 & $a a b$ & ++ & (1) & 0.2 & $a a a$ & & & 0 \\
\hline Italy & $a a a$ & & & 0 & $a a a$ & +++ & (1) & 0 & $a a a$ & & & 0 \\
\hline Japan & $a a a$ & & & 0 & $a c c$ & +++ & (2) & 0.8 & $a a b$ & +++ & (2) & 0.2 \\
\hline Mexico & $a a a$ & & & 0 & $a a c$ & +++ & (2) & 0.4 & $a a a$ & & & 0 \\
\hline Myanmar & $a a a$ & & & 0 & $a a a$ & ++ & (1) & 0 & $a a a$ & & & 0 \\
\hline Nederland & $a a a$ & & & 0 & $a a a$ & & & 0 & $a a a$ & & & 0 \\
\hline Philippines & $a a a$ & & & 0 & $a a a$ & & & 0 & $a a a$ & & & 0 \\
\hline Poland & $a a a$ & & & 0 & $a a a$ & & & 0 & $a b b$ & +++ & (1) & 0.4 \\
\hline R. of Korea & $a a a$ & & & 0 & $a c c$ & +++ & (3) & 0.8 & $c c b$ & +++ & (2) & 1 \\
\hline Russia & $a b b$ & +++ & (3) & 0.4 & $b b b$ & +++ & (4) & 0.6 & $c c b$ & +++ & (3) & 1 \\
\hline Spain & $a a a$ & & & 0 & $a a a$ & & & 0 & $a a a$ & & & 0 \\
\hline USA & $a a a$ & & & 0 & $a a a$ & + & (1) & 0 & $a a a$ & & & 0 \\
\hline Viet Nam & $c b a$ & ++ & (1) & 0.6 & $a a b$ & +++ & (1) & 0.2 & $a a a$ & & & 0 \\
\hline
\end{tabular}

MLF-index of diseases assessment in the studied countries. Score index of triad: $0.0=$ null, $0.2=$ low, $0.4=$ medium-low, $0.6=$ medium-high, $0.8=$ high, and $1.0=$ critical effect

* Semi-quantitative weigh risk of the disease with highest impact: catastrophic (+++), marketable (++), and normal (+) diseases

\# In brackets, the disease number with the indicated weight units (207) increased; consequently, the FMD triad was $c c b$. In the second period of our observation coincide with a control focused on the culled animals ("stamping-out"), which proved to be ineffective for the morbidity increased by three orders of magnitude, and led to the inclusion of several new control measures, vaccination, quarantine, destruction of animal

Table 4 Diseases assessment with MLF-index and score on producers' countries in the three periods

\begin{tabular}{|c|c|c|c|c|c|c|}
\hline \multirow[t]{2}{*}{ Disease } & \multicolumn{2}{|c|}{ 2005-2008 } & \multicolumn{2}{|c|}{ 2009-2013 } & \multicolumn{2}{|c|}{ 2014-2018 } \\
\hline & $\begin{array}{l}\text { MLF- } \\
\text { index }\end{array}$ & Score & $\begin{array}{l}\text { MLF- } \\
\text { index }\end{array}$ & Score & $\begin{array}{l}\text { MLF- } \\
\text { index }\end{array}$ & Score \\
\hline ASF & $b b b$ & 0.6 & $c c b$ & 1 & $b b b$ & 0.6 \\
\hline FMD & $a a b$ & 0.2 & $a b c$ & 0.6 & $a a a$ & 0 \\
\hline PRRS & $c b b$ & 0.8 & $a a b$ & 0.2 & $a a a$ & 0 \\
\hline CSF & $a a b$ & 0.2 & $a a a$ & 0 & $a a b$ & 0.2 \\
\hline
\end{tabular}

MLF-index assessment of the four diseases in the studied countries. Score index: $0.0=$ null, $0.2=$ low, $0.4=$ low-medium, $0.6=$ medium-high, $0.8=$ high, and $1.0=$ critical effect products, control of wildlife reservoirs, among others. The fatality decreased, impacting herd-losses; although still at a critical level, the death toll is lower. The official report shows that by 2014, the pigs slaughtered dropped to 2009, with the help of "Stamping out" strategies (QIA 2014). We note that these strategies contained the geographical spread of the outbreak but did not translate into a significant reduction in the disease' manifestation in real terms. Contrarily, Japan in the second period had an incidence of two catastrophic diseases (FMD and influenza), with 174,000 animals killed by FMD (193 cases), affecting 81 epidemiological units, with a triad $a c c$, corresponding to our category of "alarm." With no reported FMD outbreaks for the third period, there were only CSF reports, with 610 dead or slaughtered animals and two epidemiological units affected, an $a a b$ triad, which corresponds to the "safe" category. These changes in the triad and categories, in both periods, show efficient control strategies in controlling dissemination, which remained at the " $a$ " level. Fatality decreased, as in Korea, but due to the low incidence, resulted also in low herd-losses. 

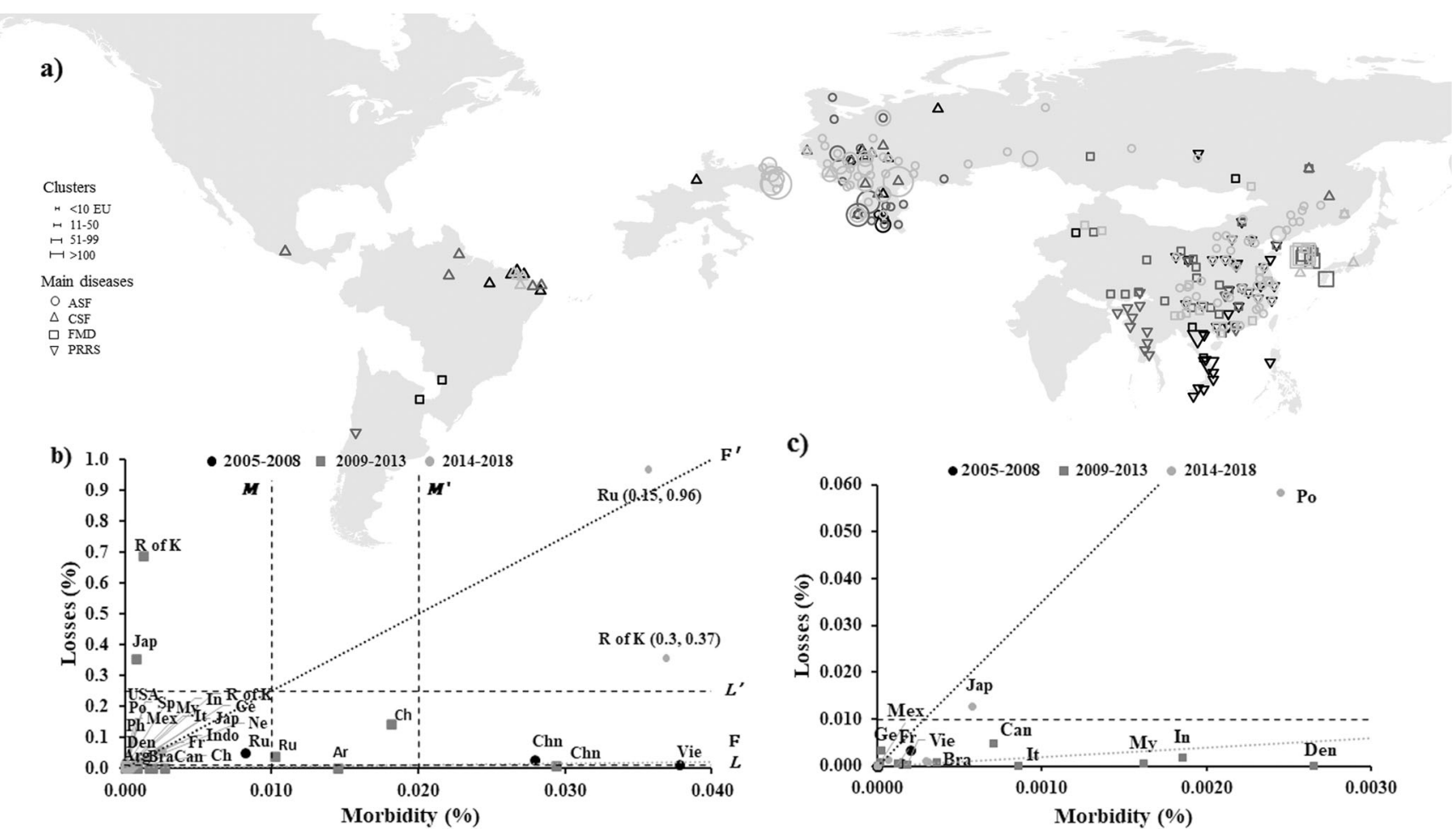

Fig. 4 a Spatiotemporal mapping (clusters of epidemiological units = EU) affected by the four main diseases in twenty-one countries in 2005-2008, 2009-2013, and 2014-2018; location is shown in the graph with respect to mortality of Korea and Russia in the second period, because of its very high morbidity rates. b Graphic representation of the multidimensional assessment; Black symbols correspond to first period, dark gray to the second period, and light gray

to the third period. $\mathbf{c}$ Blown up of data $<0.003,0.065$. For location purposes, Russia is presented among Asian countries. Ar Argentina, $\mathrm{Br}$ Brazil, Can Canada, Ch Chile, Chn China, Den Denmark, Fr France, Ge Germany, In India, It Italy, Jap Japan, Mex Mexico, My Myanmar, Ne Netherlands, Ph Philippines, Po Poland, R of K Republic of Korea, Ru Russian Federation, Sp Spain, USA United States, and Vie Vietnam. Artwork made with software R

The triad, together with the four-alarm categories proposed here (secure, preventive, alert, and alarm), is congruent with other disease evaluations. In temporal evaluations, CSF $(a a b)$, FMD ( $a a b$ ), and PRRS ( $a a a$ ) in the third period correspond to

the secure category, congruent with the stable state, characteristic of endemicity reported by other authors (European Food Safety Authority 2009), who found less severity, outbreaks reduction, and less virulent strains (Lange et al. 2012;

Table 5 Alert categories and disease tendencies in swine health

\begin{tabular}{|c|c|c|}
\hline $\begin{array}{l}\text { Alert } \\
\text { categories }\end{array}$ & MLF-index & Characteristics \\
\hline Secure & $\begin{array}{l}a a a, a a b, a a c, a b c \\
\quad a c c\end{array}$ & $\begin{array}{l}\text { Low virulence diseases and constant presence. With small outbreaks of considerable damage, constantly focused in } \\
\text { a specific region, with good disease control and good biosecurity, their economic effect does not exceed the } \\
\text { producer's capacity. }\end{array}$ \\
\hline Preventive & $a b b, b a a, b a b$ & $\begin{array}{l}\text { Diseases with moderate virulence and wide dissemination, mainly with marketable outbreaks of medium to high } \\
\text { morbidity with low losses, at the regional or national level. Its regular control and biosecurity have failed to } \\
\text { eradicate some diseases. Its economic damage involves agricultural capacity, national policy, and market tools. }\end{array}$ \\
\hline Alert & $b b b, c a a, c b a$ & $\begin{array}{l}\text { High virulence, emergent, and concomitant diseases. Outbreaks may begin in a specific area and reach a wider } \\
\text { population by unexpectedly increasing the occurrence. Standard control and biosafety measures are not sufficient } \\
\text { to contain the outbreak. Private insurers and the government can absorb economic damage. }\end{array}$ \\
\hline Alarm & $\begin{array}{l}b c c, b c b, c b b, c c a \\
\quad c c b, c c c\end{array}$ & $\begin{array}{l}\text { Epizootics with high virulence and catastrophic impact. May induce infrequent outbreaks, of international scale. } \\
\text { Commonly, these diseases exceed the control and biosecurity measures, causing alarm in the consumer and } \\
\text { disruption in the markets. The government contributes to assistance policies for the economic and social } \\
\text { significant effects, but these diseases conduce to large losses in the regional environment, seriously affecting } \\
\text { small producers. }\end{array}$ \\
\hline
\end{tabular}

Categories and characteristics according to, and modified of, $\operatorname{OCDE}(2011,2012)$; Thrusfield et al. (2018); and Hoinvile (2011) 
Stenfeldt et al. 2016), probably due to improvements in biosecurity, vaccination, and effective diagnosis (Lange et al. 2012; Arruda, 2017; Vilalta et al. 2017). With higher levels in cases, spread, and fatality, alert states characterize countries like Russia $(b b b)$ and Chile (PRRS $=b b b)$ in the second period. As previously documented, these warning manifestations are related to temporality, population size, and high density, which influenced CSF viral survival (Artois et al. 2002) in analyzed outbreaks, as well as in the spread of PRRS (Vilalta et al. 2017) sometimes with mutable forms of viruses. Russia had a "preventive" state in the first period, raised to "alert" in the second and became to "alarm" $(c c b)$ in the third, because ASF produced widespread alarm, causing economic and social problems in some countries of Asia, spreading from rural areas, villages, and later to the backyard and largescale farms. Finally, it is necessary to say that our alert categories differ from other proposed patterns of disease occurrence (Hoinville et al. 2013), mainly in that not only describes the dissemination and frequency but provides outbreak characteristics and trends according to the combinations observed in the diseases index and countries analyzed. Those results show the index utility and simplicity in alert categories for different sectors and stakeholders in two ways, (1) by suggesting disease tendencies or progressions and (2) by hinting at control measures by region or country, providing a semi-qualitative assessment. In that sense, our assessment is both spatial and temporal. With the alert categories presented here, the disease analysis could help in decision-making, prevention, and control.

Our index seeks to provide a rapid assessment based on easily accessible data. It is limited in more than one direction sense: it refers only to domestic swine, infectious diseases, notifiable diseases, and excludes productive issues like newborn and post-weaned. Also, our analysis assesses to swineherd population without age stratification, based on the precedents of risk analysis and dissemination of ASF, CSF, and PRRS, which have grouped domestic and feral pigs, including different age groups, climates, and pig densities (De la Torre et al. 2013; Porphyre et al. 2016; Arruda et al. 2017).

In conclusion, this study showed the utility that the MLFindex provides for temporal analysis of swine diseases and the benefits it offers on the impact measure of outbreaks at the national and regional scale. Criticality levels for morbidity and fatality give a simplified but plausible criterion that facilitates evaluations with large or small data. This multidimensional assessment may help decrease disease severity by providing a qualitative-quantitative evaluation of the affectation and their characteristics and, eventually, help prevent epidemics and pandemics in the decision-making and planning processes for pig-health policy. MLF-index is a complementary measure for surveillance programs, being possible to assess the epidemic progression and effectiveness of control measures. Our work demands a continuous reviewing of the thresholds, be tested in local, small, and medium farms, a form to communicate the index to the non-specialist, and a detailed comparison with a risk analysis of disease for further improvement.

Funding This work was supported by the CONACyT-Mexico (445208/ 337849-AZC).

\section{Compliance with ethical standards}

Statement of animal rights This article does not contain any studies with animal participants performed by authors.

Conflict of interest The authors declare that they have no conflicts of interest.

\section{References}

Allepuz, A., Casal, J., Pujols, J., Jové, R., Selga, I., Porcar, J. and Domingo, M., 2007. Descriptive epidemiology of the outbreak of classical swine fever in Catalonia (Spain), 2001/02 Veterinary Record, 160, 398-403

Arruda, A.G., Alkhamis, M.A., VanderWaal, K., Morrison, R.B. and Perez, A.M., 2017. Estimation of Time-Dependent Reproduction Numbers for Porcine Reproductive and Respiratory Syndrome across Different Regions and Production Systems of the US Frontiers in Veterinary Science, 4, 1-9

Artois, M., Depner, K.R., Guberti, V., Hars, J. and Rossi, S., 2002. Classical swine fever (hog cholera ) in wild boar Scientific and Technical Review International Office of Epizootics, 21, 287-303

Australian Animal Health Council, 2016. Government and livestock industry cost sharing deed in respect of emergency animal disease responses, (Australia)

Bisdorff, B., Schauer, B. and Taylor, N., 2017. Active animal health surveillance in European Union Member States : gaps and opportunities Epidemiology and Infection, 802-817

Bivand, R., Keitt, T. and Rowlingson, B., 2019. rgdal: Bindings for the "Geospatial" Data Abstraction Library

Brunsdon, C. and Chen, H., 2014. GISTools: Some further GIS capabilities for $\mathrm{R}$

Charerntantanakul, W., 2012. Porcine reproductive and respiratory syndrome virus vaccines: Immunogenicity, efficacy and safety aspects World Journal of Virology, 1, 23

Chenais, E., Boqvist, S., Emanuelson, U., von Brömssen, C., Ouma, E., Aliro, T., Masembe, C., Ståhl, K. and Sternberg-Lewerin, S., 2017. Quantitative assessment of social and economic impact of African swine fever outbreaks in northern Uganda Preventive Veterinary Medicine, 144, 134-148 (Elsevier B.V.)

De la Torre, A., Bosch, J., Iglesias, I., Muñoz, M.J., Mur, L., MartínezLópez, B., Martínez, M. and Sánchez-Vizcaíno, J.M., 2013. Assessing the risk of african swine fever introduction into the european union by wild boar Transboundary and Emerging Diseases, 62, 272-279

Dehove, A., Commault, J., Petitclerc, M., Teissier, M. and Macé, J., 2012. Economic analysis and costing of animal health: A literature review of methods and importance OIE Revue Scientifique et Technique, $31,591-617$

European Food Safety Authority, 2009. Control and eradication of Classic Swine Fever in wild boar EFSA, 7, 932 (European food safety authority)

FAO, 2007. Gridded livestock of the world, G. R. W. Wint and T. P. Robinson (eds), (FAO: Rome) 
FAOSTAT, 2019. Food and agriculture data. In: Food and agriculture Organization of the United Nations. http://www.fao.org/faostat/en/\# data/QA

Gilbert, M., Conchedda, G., Van Boeckel, T.P., Cinardi, G., Linard, C., Nicolas, G., Thanapongtharm, W., D'Aietti, L., Wint, W., Newman, S.H. and Robinson, T.P., 2015. Income disparities and the global distribution of intensively farmed chicken and pigs PLoS ONE, 10, $1-14$

Gizaw, S., Desta, H., Alemu, B., Tegegne, A. and Wieland, B., 2020. Importance of livestock diseases identified using participatory epidemiology in the highlands of Ethiopia Tropical Animal Health and Production, doi: https://doi.org/10.1007/s11250-019-02187-4 (Tropical Animal Health and Production)

Harrell Jr, F.E. and with contributions from Charles Dupont and many others, 2019. Hmisc: Harrell Miscellaneous

Hoinville, L., Alban, L., Drewe, J.A., Gibbens, J.C., Gustafson, L., Häsler, B., Saegerman, C., Salman, M. and K.D. Stärk, 2013. Proposed terms and concepts for describing and evaluating animal-health surveillance systems Preventive Veterinary Medicine, Oct 1, 1-12

Holtkamp, D.J., Kliebenstein, J.B., Neumann, E.J., Zimmerman, J.J., Rotto, H.F., Yoder, T.K., Wang, C., Yeske, P.E., Mowrer, C.L. and Haley, C.A., 2013. Assessment of the economic impact of porcine reproductive and respiratory syndrome virus on United States pork producers Journal of Swine Health and Production, 21, 72-84

Jung-hyang, S., 2019. How far can African swine fever spread? Journal Of Veterinary Science, 20, 1-5

Jurado, C., Mur, L., Pérez Aguirreburualde, M.S., Cadenas-Fernández, E., Martínez-López, B., Sánchez-Vizcaíno, J.M. and Perez, A., 2019. Risk of African swine fever virus introduction into the United States through smuggling of pork in air passenger luggage Scientific Reports, 9, 1-7

Lange, M., Kramer-schadt, S., Blome, S., Beer, M. and Thulke, H., 2012. Disease severity declines over time after a wild boar population has been affected by classical swine fever - Legend or actual epidemiological process ? Preventive Veterinary Medicine, 106, 185-195 (Elsevier B.V.)

Morilla, A., 2003. Las enfermedades virales emergentes de los cerdos Ciencias Veterinarias, 197-227

Mur, L., Martínez-López, B. and Sánchez-Vizcaíno, J.M., 2012. Risk of African swine fever introduction into the European Union through transport-associated routes: returning trucks and waste from international ships and planes BMC Veterinary Research, 8

Mur, L., Martínez-López, B., Costard, S., de la Torre, A., Jones, B.A., Martínez, M., Sánchez-Vizcaíno, F., Muñoz, M.J., Pfeiffer, D.U., Sánchez-Vizcaíno, J.M. and Wieland, B., 2014. Modular framework to assess the risk of African swine fever virus entry into the European Union BMC Veterinary Research, 10, 1-13

Nthiwa, D., Alonso, S., Odongo, D., Kenya, E. and Bett, B., 2019. A participatory epidemiological study of major cattle diseases amongst Maasai pastoralists living in wildlife-livestock interfaces in Maasai Mara, Kenya Tropical Animal Health and Production, 51, 10971103 (Tropical Animal Health and Production)

OECD, 2011. Managing Risk in Agriculture Managing Risk in Agriculture: Policy Assessment and Design, 255 (OECD Publishing)
OIE, 2011. Terrestrial Animal Health Code, Twentieth (Paris, France)

OIE, 2019. Exceptional epidemiological events. In: WAHIS Interface. https://www.oie.int/wahis_2/public/wahid.php/ Countryinformation/Countryreports

OIE, 2020. African Swine Fever (ASF). Report No. 47: 2016-2020,

Perez, S., Brihn, A., Perez, A., Torrison, J., Torremorell, M., Corzo, C., Sundberg, P. and Deen, J., 2019. Swine Disease Global Surveillance Report Swine Information Center of University of Minnesota, July 1-Aug, 1-10 (Minnesota)

Perry, B., Mcdermott, J. and Randolph, T., 2001. Can epidemiology and economics make a meaningful contribution to national animaldisease control ? 48

Porphyre, T., Boden, L.A., Correia-Gomes, C., Auty, H.K., Gunn, G.J. and Woolhouse, M.E.J., 2016. Using national movement databases to help inform responses to swine disease outbreaks in Scotland: The impact of uncertainty around incursion time Scientific Reports, 6, 19 (Nature Publishing Group)

QIA, 2014. FMD situation \& Control Strategy in Korea Contents, Park Sungdae D.V.M. Veterinary Epidemiology Division Animal and Plant Quarantine Agency.

R Core Team, 2013. R: A language and environment for statistical computing. (R Foundation for Statistical Computing: Vienna, Austria)

Rushton, J. and Gilbert, W., 2016. Economía de la sanidad animal: costos directos e indirectos de los brotes de enfermedades animales, $84 \mathrm{SG} /$ 9 (Royal Veterinary College, Londres, Reino Unido: París)

Sánchez-Vizcaíno, J.M. ; Mur, L.; Gomez-Villamandos, J.C.. and Carrasco, L., 2015. An Update on the Epidemiology and Pathology of African Swine Fever Journal of Comparative Pathology, 152, 9-21

Stabler, B., 2013. shapefiles: Read and Write ESRI Shapefiles

Stenfeldt, C., Segundo, F.D.S., de los Santos, T., Rodriguez, L.L. and Arzt, J., 2016. The pathogenesis of foot-and-mouth disease in pigs Frontiers in Veterinary Science, 3, 1-12

Tousignant, S.J., Perez, A.M., Lowe, J.F., Yeske, P.E. and Morrison, R.B., 2015. Temporal and spatial dynamics of PRRSV infection in the United States American Journal of Veterinary Research, 76, 70 76

VanderWaal, K. and Deen, J., 2018. Global trends in infectious diseases of swine 2018

Verzani, J., 2018. UsingR: Data Sets, Etc. for the Text "Using R for Introductory Statistics", Second Edition

Vilalta, C., Arruda, A.G., Tousignant, Steven J.P., Valdes-Donoso, P., Muellner, P., Muellner, U., Alkhamis, M.A., Morrison, R.B. and Perez, A.M., 2017. A review of quantitative tools used to assess the epidemiology of porcine reproductive and respiratory syndrome in U.S. swine farms using Dr. Morrison's Swine Health Monitoring Program Data Frontiers in Veterinary Science, 4, 1-7

World Bank, TAFS forum, OIE and FAO, 2011. World Livestock Disease Atlas. A Quantitative Analysis of Global Animal Health Data (2006-2009),

Zhou, L. and Yang, H., 2010. Porcine reproductive and respiratory syndrome in China Virus Research, 154, 31-37 (Elsevier B.V.)

Publisher's note Springer Nature remains neutral with regard to jurisdictional claims in published maps and institutional affiliations. 